\title{
Recent Advances in Attribute-Based Signatures
}

\author{
Yusuke Sakai \\ yusuke.sakai@aist.go.jp \\ National Institute of Advanced Industrial Science and Technology \\ Tokyo, Japan
}

\begin{abstract}
Attribute-based signatures [11] are an advanced signature primitive that allows a signer to prove the possession of attributes with minimum level of disclosure of his privacy. In particular, by issuing a signature, the singer can prove the possession of a set of attributes that satisfies some public policy, while hiding any further information on the attributes beyond the fact that the attributes satisfy the public policy. This primitive has many interesting applications, for example, anonymous credentials [16], attribute-based messaging [11], and secret leaking [11].

There are two main performance measures of attribute-based signatures. The first is expressiveness of the policy, namely, the computational class with which the policy is described, while the other is the computational overhead, for example, the signature size. While initial schemes mainly tired to investigate a trade-off between expressiveness and computational overhead [1, 6, 8-10,16], recently the research moves to aiming at achieving very expressive policy. Such constructions includes the construction for non-monotone span programs as the policy [12], those for unbounded circuits from bilinear maps [14] from lattices in the random oracle model [5], and from lattices in the standard model [17], those for Turing machines and nondeterministic finite automata [15], and that for unbounded arithmetic branching programs [2].

Another direction in this research area is to extend the functionality of the attribute-based signature primitive. This direction includes constructing traceable attribute-based signatures [6], decentralized attribute-based signatures [13], decentralized traceable attribute-based signatures $[4,7]$, and hierarchical attribute-based signatures [3].

In this talk, we give a brief overview of the recent advances in the area of attribute-based signatures, pick up some of the recent schemes, and introduce the key techniques for these constructions.
\end{abstract}

\section{CCS CONCEPTS}

- Security and privacy $\rightarrow$ Digital signatures.

\section{KEYWORDS}

digital signatures, anonymous authentication, attribute-based signatures

Permission to make digital or hard copies of all or part of this work for personal or classroom use is granted without fee provided that copies are not made or distributed for profit or commercial advantage and that copies bear this notice and the full citation on the first page. Copyrights for components of this work owned by others than ACM must be honored. Abstracting with credit is permitted. To copy otherwise, or republish, to post on servers or to redistribute to lists, requires prior specific permission and/or a fee. Request permissions from permissions@acm.org.

APKC '19, fuly 8, 2019, Auckland, New Zealand

(ㄷ) 2019 Association for Computing Machinery.

ACM ISBN 978-1-4503-6784-4/19/07 . \$ \$15.00

https://doi.org/10.1145/3327958.3329545

\section{ACKNOWLEDGMENTS}

A part of this work was supported by JST CREST Grant Number JPMJCR19F6, Japan and JSPS KAKENHI Grant Number 18K18055, Japan .

\section{REFERENCES}

[1] Cheng Chen, Jie Chen, Hoon Wei Lim, Zhenfeng Zhang, Dengguo Feng, San Ling, and Huaxiong Wang. 2013. Fully Secure Attribute-Based Systems with Short Ciphertexts/Signatures and Threshold Access Structures. In CT-RSA 2013. LNCS, Vol. 7779. Springer Berlin Heidelberg, 50-67.

[2] Pratish Datta, Tatsuaki Okamoto, and Katsuyuki Takashima. 2019. Efficient Attribute-Based Signatures for Unbounded Arithmetic Branching Programs. In PKC 2019, Dongdai Lin and Kazue Sako (Eds.). Lecture Notes in Computer Science, Vol. 11442. Springer International Publishing, 127-158.

[3] Constantin-Cătălin Drăgan, Daniel Gardham, and Mark Manulis. 2018. Hierarchical Attribute-Based Signatures. In CANS 2018, Jan Camenisch and Panos Papadimitratos (Eds.). Vol. 11124. Springer International Publishing, 213-234.

[4] Ali El Kaafarani, Essam Ghadafi, and Dalia Khader. 2014. Decentralized Traceable Attribute-Based Signatures. In CT-RSA 2014. LNCS, Vol. 8366. Springer Berlin Heidelberg, 327-348.

[5] Ali El Kaafarani and Shuichi Katsumata. 2018. Attribute-Based Signatures for Unbounded Circuits in the ROM and Efficient Instantiations from Lattices. In PKC 2018 (LNCS), Vol. 10770. Springer International Publishing, 89-119.

[6] Alex Escala, Javier Herranz, and Paz Morillo. 2011. Revocable Attribute-Based Signatures with Adaptive Security in the Standard Model. In AFRICACRYPT 2011. LNCS, Vol. 6737. Springer Berlin Heidelberg, 224-241.

[7] Essam Ghadafi. 2015. Stronger Security Notions for Decentralized Traceable Attribute-Based Signatures and More Efficient Constructions. In CT-RSA 2015. LNCS, Vol. 9048. Springer Berlin Heidelberg, 391-409.

[8] Javier Herranz, Fabien Laguillaumie, Benoît Libert, and Carla Ràfols. 2012. Short Attribute-Based Signatures for Threshold Predicates. In CT-RSA 2012. LNCS, Vol. 7178. Springer Berlin Heidelberg, 51-67.

[9] Jin Li, Man Ho Au, Willy Susilo, Dongqing Xie, and Kui Ren. 2010. Attributebased Signature and Its Applications. In Proceedings of the 5th ACM Symposium on Information, Computer and Communications Security. ACM, 60-69.

[10] Jin Li and Kwangjo Kim. 2008. Attribute-Based Ring Signatures. Cryptology ePrint Archive, Report 2008/394. http://eprint.iacr.org/.

[11] Hemanta K. Maji, Manoj Prabhakaran, and Mike Rosulek. 2011. Attribute-Based Signatures. In CT-RSA 2011. LNCS, Vol. 6558. Springer Berlin Heidelberg, 376392.

[12] Tatsuaki Okamoto and Katsuyuki Takashima. 2011. Efficient Attribute-Based Signatures for Non-monotone Predicates in the Standard Model. In PKC 2011. LNCS, Vol. 6571. Springer Berlin Heidelberg, 35-52.

[13] Tatsuaki Okamoto and Katsuyuki Takashima. 2013. Decentralized AttributeBased Signatures. In PKC 2013. LNCS, Vol. 7778. Springer Berlin Heidelberg, 125-142.

[14] Yusuke Sakai, Nuttapong Attrapadung, and Goichiro Hanaoka. 2016. AttributeBased Signatures for Circuits from Bilinear Map. In PKC 2016. LNCS, Vol. 9614. Springer Berlin Heidelberg, 283-300.

[15] Yusuke Sakai, Shuichi Katsumata, Nuttapong Attrapadung, and Goichiro Hanaoka. 2018. Attribute-Based Signatures for Unbounded Languages from Standard Assumptions. In ASIACRYPT 2018, Thomas Peyrin and Steven Galbraith (Eds.). Lecture Notes in Computer Science, Vol. 11273. Springer International Publishing, 493-522.

[16] Siamak F. Shahandashti and Reihaneh Safavi-Naini. 2009. Threshold AttributeBased Signatures and Their Application to Anonymous Credential Systems. In AFRICACRYPT 2009. LNCS, Vol. 5580. Springer Berlin Heidelberg, 198-216.

[17] Rotem Tsabary. 2017. An Equivalence Between Attribute-Based Signatures and Homomorphic Signatures, and New Constructions for Both. In TCC 2017. LNCS, Vol. 10678. Springer International Publishing, 489-518. 